\title{
The Diminishing Precision of Memory for Time
}

\author{
John E. Scofield and Jeffrey D. Johnson \\ University of Missouri
}

Correspondence should be addressed to either author at:

Department of Psychological Sciences

University of Missouri

Columbia, MO 65211 USA

jel7c5@mail.missouri.edu (J.E.S.) or jeffreyjohnson@missouri.edu (J.D.J)

Length (Introduction, Results, Discussion): 3,505 words

Number of figures: 2

\begin{abstract}
Knowing when an event took place can provide several benefits to episodic memory, such as distinguishing among multiple traces, learning sequences of events, and guiding a search strategy. As a tool for understanding memory, time is particularly appealing given its everchanging quality, the constant possibility to associate it with encoded events, and the ease with which it can be targeted at retrieval. Whereas studies of episodic retrieval typically employ categorical and probabilistic measures of retrieval success, characterizing a continuous feature such as time warrants measures particularly sensitive to the fidelity, or precision, of retrieved information. Here, we adapt a paradigm for assessing the fine-grained precision of retrieval to understand the nature of judging the time at which a memory was encoded. Subjects studied a series of pictures and then undertook a test in which they placed each picture, as precisely as possible, along a continuous timeline representing the study list. Based on mixture-modeling analyses of the test response errors, the primary results were that temporal judgments were less accurate with passing time, and this change was due to diminished precision as opposed to an increased rate of guessing. Moreover, although we observed a negligible influence of guessing, subjects exhibited a clear effect of bias that favored recent responses. Together, in contrast to numerous studies of memory for other continuous features (e.g., color and location), our findings demonstrate a novel pattern of decision factors, suggesting that the retrieval of time might highlight distinct attributes of episodic memory.
\end{abstract}

Keywords: episodic memory; temporal memory; mixture-modeling; retrieval; recollection 


\section{The Diminishing Precision of Memory for Time}

Episodic memory allows the unique events comprising our personal history to be encoded and retrieved. The uniqueness of such event traces is afforded by several factors, including the combination of details bound to each event, and the context that places the event in space and time. Of these, time is particularly interesting given that it is a ubiquitous and ever-changing feature of our experiences, and for its relevance to a range of memory phenomena such as forgetting, sequential learning, and event segmentation (for general reviews, see Friedman, 1993; Eichenbaum, 2013; Ranganath \& Hsieh, 2016; Howard, 2018). However, aside from an abundance of studies examining recency judgments and time-related changes in traces, little is known about the qualitative characteristics of the temporal information associated with episodic memories. The current study employs a mixture-modeling approach with continuous behavioral measures to elucidate the nature of temporal retrieval. In particular, we address questions about how precisely temporal judgments are made, whether precision changes with passing time, and the roles of other decisional factors (i.e. guessing and bias) in such judgments.

One barrier to investigating a continuous feature such as time is that memory paradigms often focus only on categorical distinctions regarding the basis for retrieval, as is the case for example with the remember/know task in distinguishing detailed recollection from acontextual familiarity (for reviews, see Yonelinas, 2002; Skinner \& Fernandes, 2007). While recent iterations of this task have advanced to graded measures of familiarity (e.g., Yonelinas, Otten, Shaw, \& Rugg, 2005; Montaldi, Spencer, Roberts, \& Mayes, 2006), investigations of the continuous nature of recollection are less common, with notable exceptions dealing with the amount of retrieval (e.g., Wilding, 2000; Vilberg \& Rugg, 2007) and levels of source memory confidence (e.g., Mickes, Wais, \& Wixted, 2009; Hayes, Buchler, Stokes, Kragel, \& Cabeza, 2011). An alternative paradigm, popularized in working memory research (for review, see Ma, Husain, \& Bays, 2014), has been used to assess the precision of memory retrieval. In the context of long-term memory, a common version of this paradigm has subjects encode words associated with spatial positions on a circle and then later, given the word cue, denote the correct position as precisely as possible (Harlow \& Donaldson, 2013; Murray, Howie, \& Donaldson, 2015; Harlow \& Yonelinas, 2016). Behavioral responding in such a task can be analyzed with a mixturemodeling approach in which the precision of retrieval varies continuously and can be dissociated from the involvement of other behavioral factors, such as guessing and response bias, as well as from other neural correlates of retrieval success (e.g., Richter, Cooper, Bays, \& Simons, 2016).

We sought to extend the precision paradigm described above to further understand the retrieval of continuous temporal information. Whereas several classic memory studies have had subjects estimate the time at which items were previously studied, such judgments were often relatively coarse in that they distinguished between multiple study lists or across sizable portions of a single list (e.g., Hintzman \& Block, 1971; Hintzman, Block, \& Summers, 1973; McCormack, 1984). In a recent study advancing the earlier work, Montchal, Reagh, \& Yassa (2019) had subjects watch a half-hour television show and then later place still-frames along a timeline corresponding to their initial occurrence. Although the timeline had a higher resolution than the previous studies, thereby allowing for better precision estimates, subjects in a control condition who did not initially watch the show also exhibited some degree of precision. This is not surprising given the natural progression of the show's plot and the possible presence of other informative cues. We eliminated such cues here by employing a study phase consisting of a 
series of randomly-ordered and equally-spaced pictures, with no breaks to serve as potential landmarks and no instructions referencing the temporal aspect of the later memory test. Our approach is similar to that of another recent study, by Jenkins and Ranganath (2010), in which subjects were presented with a list of pictures in the context of a working memory task and were subsequently given a test that involved placing each picture on a timeline corresponding to the study list. The authors observed that temporal response error was smaller for items studied more recently compared to remotely (also see Lositsky et al., 2016). By implementing a mixturemodeling approach, we first estimated the precision of temporal judgments and whether it diminished with passing time (as predicted by the foregoing results). Moreover, this approach allowed us to novelly test for the presence of other decision-making (i.e. non-mnemonic) factors, such as guessing and response bias, and consequently, to rule out those factors in accounting for any changes in temporal memory (also see Brady, Konkle, Gill, Oliva, \& Alvarez, 2013).

\section{Methods}

\section{Subjects}

Thirty-two University of Missouri (MU) students participated for partial course credit. Our sample size was chosen to be comparable to that in previous studies using mixture modeling for long-term memory performance (e.g., Murray et al., 2015; Harlow \& Yonelinas, 2016). Inclusion criteria were: 18-30 years old, native-English speaking, and no history of neurological disease. Informed consent was obtained in accordance with the MU Institutional Review Board. Data were initially screened to confirm each subject's active engagement with the task, defined as moving the computer mouse from the starting position on $>95 \%$ of test trials (see Harlow \& Yonelinas, 2016). Two subjects were excluded by this criterion, with an additional subject removed for having a high percentage $(28 \%)$ of fast $(<500 \mathrm{~ms})$ temporal judgments. The final sample of subjects (20 females, 9 males) were $18-22$ years old $(M=19.1, S D=1.1)$.

\section{Stimuli and Procedure}

The stimulus pool consisted of 300 color pictures of common, nameable objects. Each picture subtended a visual angle of about $3.1^{\circ}$ and was presented centrally on a light gray ( $70 \%$ white) background. Stimuli were displayed on a 24 -inch widescreen LCD monitor $(1024 \times 768$ resolution) viewed at a distance of approximately 1 meter. Stimulus presentation was controlled with the Cogent2000 toolbox (v.1.32; http://www.vislab.ucl.ac.uk) in MATLAB (R2012a; MathWorks, Natick, MA).

The experimental session ( 45 minutes) consisted of a study phase followed by a test phase. Instructions and practice on the study phase were administered first, with details about the test phase withheld until the study phase concluded. Although subjects were kept naïve about the nature of the test until immediately prior to its start, it is possible that they anticipated some sort of memory test, as the experiment was advertised as a "memory study".

The study phase comprised a single block of 300 pictures, with each displayed for 3000 $\mathrm{ms}$ and followed by a central plus sign presented for $500 \mathrm{~ms}$. Subjects were informed of the number of trials and total time of the study phase (17.5 minutes) prior to its start. Subjects were instructed to rate the pleasantness of each picture on a 4-point scale (very pleasant, somewhat 
pleasant, somewhat unpleasant, and very unpleasant) via key presses mapped to their left little through index fingers. The response options were displayed at the bottom of the screen throughout the study phase.

Following the study phase, subjects received instructions and practice on the test phase, which consisted of all study pictures being presented in a randomized order. Each test trial began with a picture presented above a horizontal time scale. The time scale ranged from trial 1 on the left to trial 300 on the right, with labels marking every 50 trials. On each trial, an arrow was initially displayed at the midpoint of the scale (trial 150). Subjects were instructed to use the computer mouse to move the arrow, as precisely as possible, to the position at which they thought the picture occurred at study, and to click the left mouse button to finalize their response. A vertical confidence scale, ranging from $0 \%$ at the bottom to $100 \%$ at the top (with labels marking 20\% increments), then replaced both the picture and time scale. An arrow next to the scale was initialized at $50 \%$ on each trial, and subjects were to rate their confidence about correctly retrieving the picture's study position, irrespective of precision (also see Harlow \& Yonelinas, 2016). After the confidence judgment was made by pressing the left mouse button, a central plus sign was displayed for $1000 \mathrm{~ms}$ until the next trial began.

\section{Data Availability and Analysis}

The behavioral data and analysis scripts are available at https://osf.io/c7nge. Primary analyses were conducted using MemToolbox (v. 1.0.0; Suchow, Brady, Fougnie, \& Alvarez, 2013) in MATLAB (R2012a), with modifications made to the toolbox to model responses along a linear (time) scale. Prior to analysis, study trials on which subjects failed to respond or did so outside a predefined interval of 500-3000 ms after picture onset were removed (2.9\% of trials; $\mathrm{SD}=3.0$ ). Although the temporal position and confidence judgments during the test phase were self-paced, trials on which subjects made the temporal response faster than $500 \mathrm{~ms}$ or made either response slower than $10 \mathrm{~s}$ were removed $(2.4 \%$ of trials; $\mathrm{SD}=3.6)$. Given the infrequency of responses near the start and end of the scale, and due to the potential for temporal judgments to be affected by these boundaries, data for the first 50 and last 50 study trials were excluded from all analyses (for analogous approaches, see Montchal et al., 2019; Nilakantan, Bridge, Gagnon, VanHaerents, \& Voss, 2017; Nilakantan, Bridge, VanHaerents, \& Voss, 2018).

Trials were scored in terms of error distance (in number of trials) between the actual study position of the picture and its judged position on the test. The temporal (position) scale was 600 pixels wide, providing a resolution of every .5 trial. The confidence scale was 500 pixels tall, giving a resolution of $.2 \%$. The posterior distribution of each parameter was estimated with an adaptive Markov Chain Monte Carlo (MCMC) algorithm (see Andrieu, De Freitas, Doucet, \& Jordan, 2003) in which three separate chains were run (based on different starting values) and convergence was checked after every 200 samples (Gelman \& Rubin, 1992). For the group- and subject-based models, the respective numbers of post-convergence samples collected were 4500 and 6000. Model comparison was based on Bayesian Information Criterion (BIC; Schwarz, 1978). Bayes factors (BFs) were computed with a set of MATLAB functions (Schwarzkopf, 2015), using a default scaling factor (r) of 707 (see Rouder et al., 2009; Morey, Rouder, \& Jamil, 2018). All BFs are reported as $\mathrm{BF}_{10}$, where values $>1$ indicate evidence favoring the alternative hypothesis, and positive values $<1$ indicate evidence favoring the null hypothesis. 


\section{Results}

During the study phase, the mean response time (RT) for making pleasantness judgments was $1424 \mathrm{~ms}(\mathrm{SD}=246)$. For the test phase, the mean RTs for temporal (study position) and confidence judgments were 2720 (SD = 794) and $1064 \mathrm{~ms}(\mathrm{SD}=386)$, respectively. Figure 1A displays the group-level error distribution of temporal responses. Centered on each study trial, the mean absolute error was 7.69 trials $(\mathrm{SD}=13.51)$. (As an aside, there was no relationship between error and RT of temporal judgments $[r=-.01, p=.69]$.) To further parameterize temporal precision, the error distribution was modeled by a normal function with a mean of zero and the standard deviation corresponding to precision error (hereafter, $\sigma$ ). The resulting maximum a posterior probability (MAP) estimate of $\sigma$ was 75.32 (95\% highest density interval $[\mathrm{HDI}]=[73.93,76.80])$. Modeling individual subjects gave rise to a similar level of precision error: $\mathrm{M}=74.95$ and $\mathrm{SD}=8.32$ (see Figure 1B).

\section{Temporal Precision Changes with Passing Time}

Next, we investigated whether temporal precision changes with passing time by dividing the study list in halves and applying the modeling procedure described above to the error distribution for each half. As displayed in Figures 1B and 1C, group-level precision error was higher for the first half (hereafter, remote; $\sigma=79.19$ [77.24, 81.28]) than the second half (recent; $\sigma=71.23[69.48,73.22]$ ), and subject-wise modeling confirmed this difference was significantly different (remote: $\mathrm{M}=78.59, \mathrm{SD}=11.38$; recent: $\mathrm{M}=70.21, \mathrm{SD}=11.70 ; \mathrm{t}_{28}=2.84, \mathrm{p}=.008$, $\mathrm{BF}_{10}=3.78$ ). Closer examination of the temporal response distributions revealed that precision error was not only lower for recent compared to remote trials, but was considerably lower for the middle of the study list. Figure 1B displays this pattern for trials binned into quartiles. To better isolate the remote-recent effect from the difference in precision for the middle versus extreme quartiles, the subject-wise $\sigma$ estimates of the quartiled data were submitted to a 2 (remote, recent) $\times 2$ (middle, end) ANOVA. The analysis revealed main effects of both factors (respectively, $\mathrm{F}_{1,28}$ $=8.72$ and 227.56, $\mathrm{p}=.006$ and $\mathrm{p}<.001)$ but no interaction $(\mathrm{F}<1)$. Planned contrasts confirmed that remote trials were associated with lower precision for the first compared to fourth quartile $\left(\mathrm{M}=88.60\right.$ and 79.02, $\mathrm{SD}=14.17$ and $\left.16.15 ; \mathrm{t}_{28}=2.20, \mathrm{p}=.036, \mathrm{BF}_{10}=1.57\right)$ and for the second versus third quartile $\left(\mathrm{M}=66.78\right.$ and $59.75, \mathrm{SD}=10.63$ and $9.50 ; \mathrm{t}_{28}=3.64, \mathrm{p}=.001$, $\mathrm{BF}_{10}=30.82$ ).

\section{Temporal Judgments Exhibit a Recency Bias}

By comparing the distribution of temporal errors to the corresponding model fit, as displayed in Figure 1A, it becomes apparent that errors overall tended to be more recent than remote; this shift is more even more pronounced for remote trials, as shown in Figure $1 \mathrm{C}$. We therefore tested this effect by adding a bias parameter $(\mu)$, corresponding to a shift in the mean of the normal distribution, to the modeling procedure. The resulting fit of the 2-parameter $(\mu, \sigma)$ model is shown in Figure 2A, where the corresponding shift with respect to the $\sigma$-only model can be seen. The group-based $\mu$ estimate of $7.48[5.62,9.16]$ was consistent with the subject-based results $(M=7.70, S D=13.49$; see Figure $2 B)$, which were statistically greater than zero $\left(\mathrm{t}_{28}=\right.$ 3.07, $\mathrm{p}=.004, \mathrm{BF}_{10}=8.72$ ). Moreover, the estimates of $\sigma$ for the 2-parameter model resembled those of the original model (group: 74.96, [73.69, 76.32]; subjects: $\mathrm{M}=73.34, \mathrm{SD}=8.33$ ). 
Highlighting the importance of bias, despite it adding complexity, model comparison indicated that including it was preferable to excluding it ( $\mathrm{BIC}=63,502.01$ and $63,457.38$, respectively).
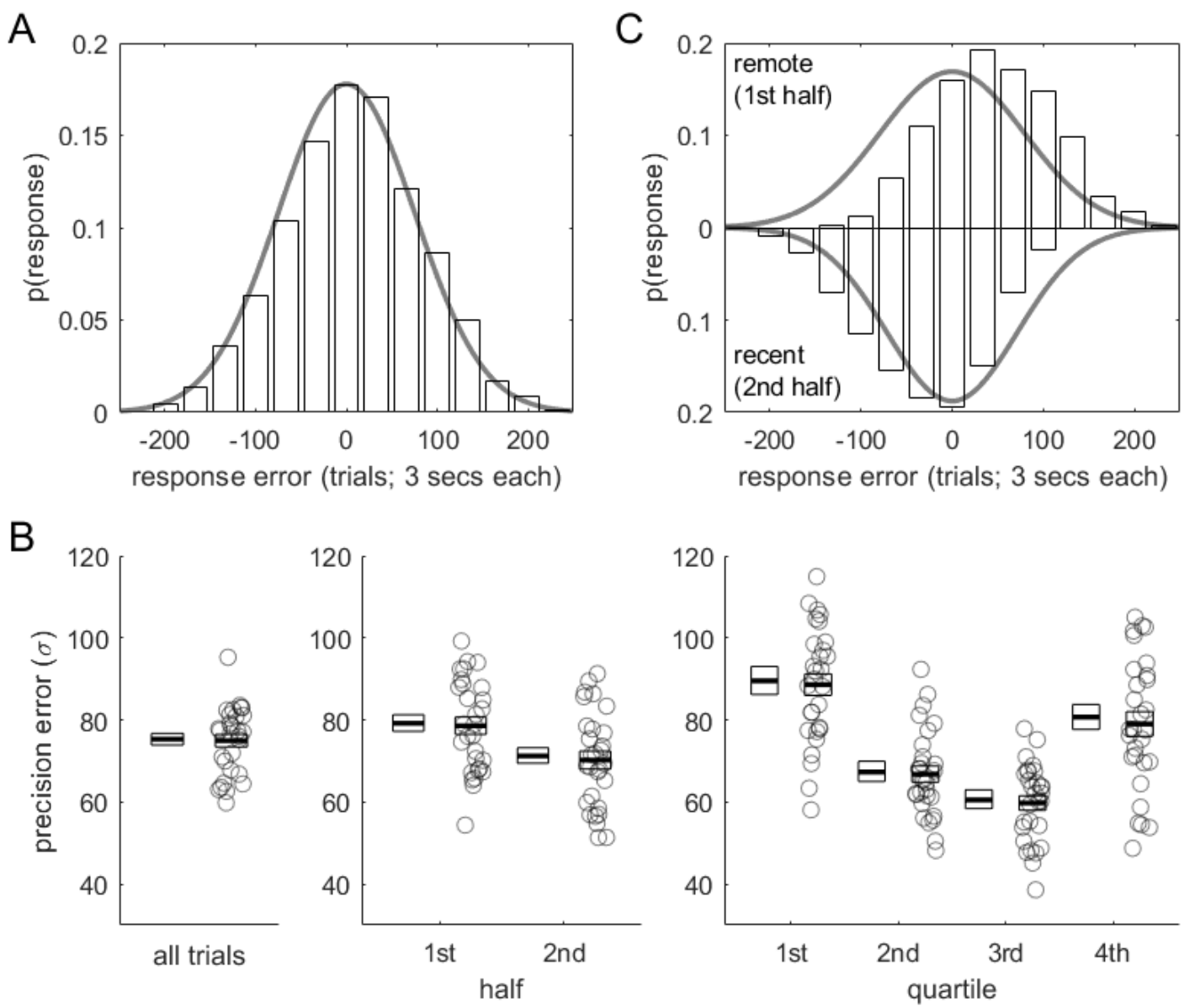

Figure 1. (A) Histogram of the group-level distribution of temporal response errors, centered (at 0 ) on each study trial. Positive/negative values respectively reflect responses that are more recent/remote than the correct position. The line corresponds to the estimated normal function of the 1-parameter (precision-only) model: $\mu=0$ and $\sigma=75.32$. (B) The precision $(\sigma)$ results for all, halved, and quartiled trials. For each pair of box plots, the left one indicates the MAP estimate and 95\% HDI for the group analysis, and the right one indicates the mean \pm SEM for the subject-wise analysis. The results for individual subjects are plotted with circles. (C) Histograms of the group-level distributions of temporal errors, separated according to the first (remote) and second (recent) halves of the study list. The line plots indicate the best-fitting normal functions for each condition $(\mu=0$; remote $\sigma=79.19$; recent $\sigma=71.23)$. Data for the recent half is arbitrarily plotted downward for clarity. 


\section{Guessing is Negligible in Temporal Judgments}

In keeping with prior studies that have often used continuous response paradigms to dissociate precision from guessing (e.g., Zhang \& Luck, 2008; Brady et al., 2013; Harlow \& Yonelinas, 2016), we next fit the temporal error distribution with a 3-parameter model that included $\sigma$ and $\mu$ (as above) and parameterized the proportion of guessing trials (hereafter, $\lambda$ ) with a uniform distribution. The estimates for precision error and bias were similar to those reported above: $\sigma=74.81[73.33,76.49](\mathrm{M}=72.69, \mathrm{SD}=8.16)$ and $\mu=7.68[5.37,9.43](\mathrm{M}=$ 7.82, SD = 13.57). Guessing, however, was near zero for both group- (5.0e-5 [5.0e-5, .009]) and subject-based models $(\mathrm{M}=.0075, \mathrm{SD}=.016)$. To further test that guessing was negligible, we directly compared the 2- and 3-parameter models, the results of which favored the former (respectively, $\mathrm{BIC}=63,457.38$ and 63,467.84).
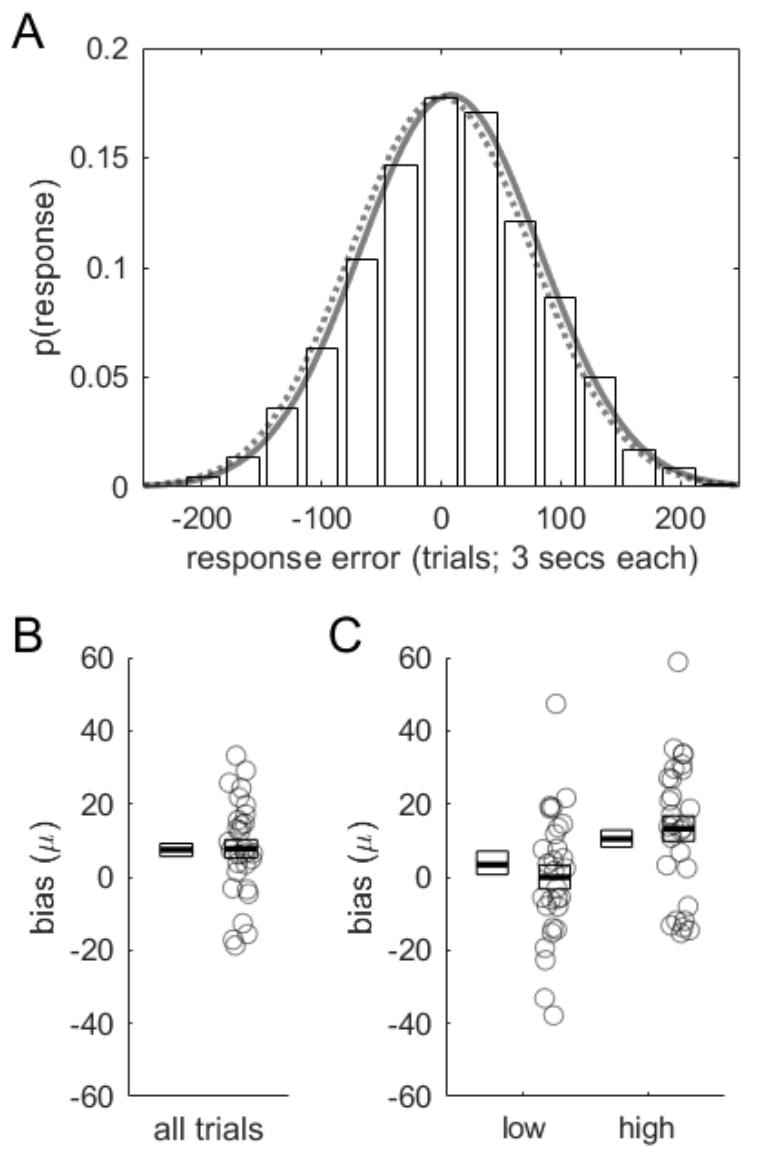

Figure 2. (A) Group-level distribution and 1parameter model fit (dotted line) of temporal response errors, as shown in Figure 1A, along with the fit of the 2-parameter model (solid line) - precision $(\sigma=74.96)$ and bias $(\mu=7.48)$. (B) Box plots indicating the group-wise (MAP, HDI) and subject-wise $(\mathrm{M} \pm \mathrm{SEM})$ results for the bias parameter, with data for individual subjects also plotted. (C) Bias parameter results corresponding to separate modeling of trials in which low vs. high confidence was designated (with a 50\% cutoff).

To ensure that any subtle differences in guessing across the study list were not missed, we additionally included it in modeling the data binned into halves. Notably, we excluded the bias parameter for these analyses, as preliminary analyses showed that it varied widely across halves of the study list (likely due to the boundaries of the study list). The $\lambda$ estimates were again near zero for both the first (1.6e-4 [1.7e-4, .014]) and second halves (3.6e-4 [4.2e-4, .015]), with the subject-wise means suggesting that there is no strong evidence for guessing rate changes 
across time (respectively, $\mathrm{M}=.0088$ and $.014, \mathrm{SD}=.020$ and $.030 ; \mathrm{t}_{28}=.73, \mathrm{p}=.47, \mathrm{BF}_{10}=$ $.73)$.

As a further test of whether our modeling approach could appropriately capture guessing across a bounded linear scale, a series of simulations were conducted in which 10,25 , or $50 \%$ of responses in the group data were randomly selected and replaced with random responses (indicative of guessing). The results and scripts for these analyses are provided at https://osf.io/c7nge, but we provide a brief summary here. In one subset of simulations, guess responses were drawn uniformly across the scale; in another subset, we accounted for subjects' tendencies to respond toward the middle of the scale by drawing randomly from a truncated normal distribution. Given that we expected the latter distribution to be wider than that corresponding to our precision judgments $(\mathrm{SD} \approx 75)$, we initially doubled it $(\mathrm{SD}=150)$ before also simulating further multiples. Based on the model comparison approach described earlier, models including the uniform guessing component were preferred in every case (for uniform guesses: $\triangle \mathrm{BIC}=32.66,135.71$, and 425.08 , for the respective 10,25 , and $50 \%$ simulations; for normally-distributed guesses at $\mathrm{SD}=150: \Delta \mathrm{BIC}=11.11,46.08$, and 129.59 , respectively). Two additional results of these analyses are worth noting. First, by the time the normal distribution was quadrupled in width (i.e. SD = 300) and the tails were truncated, the simulated guesses appeared relatively flat, converging with the findings based on the uniform distribution. Second, as would likely be predicted, the correct (simulated) proportions of guessing trials were more accurately estimated as the SD of our distribution increased, with the resulting parameters for SD $=150$ being roughly $40 \%$ of their expected sizes. Nevertheless, these findings do appear to validate the sensitivity of our modeling approach and further emphasize the negligible role of guessing in the actual data.

\section{Confidence is Associated with a Recency Bias}

Although confidence judgments were included in the design to follow previous studies (e.g., Harlow \& Donaldson, 2013; Harlow \& Donaldson, 2016), we suspected from the outset that subjects might be unable to treat them distinctly from temporal judgments, and we thus had no specific hypotheses regarding differences in confidence. For completeness, though, we summarize the confidence results and then consider them in the context of the modeling approach described above. Confidence judgments were spread considerably across the 0-100\% scale $(M=53.44, S D=9.74)$. Contrary to what might be expected if confidence tracked temporal precision, it was not correlated with the absolute value of error $(\mathrm{r}=.0088, \mathrm{p}=.51$; cf. Harlow \& Yonelinas, 2016). Nonetheless, the ratings were binned into high and low confidence (using a 50\% cutoff), and the 3-parameter $(\sigma, \mu$, and $\lambda$ ) modeling procedure was applied separately to the data from each bin. Across high versus low confidence, no decisive evidence of any differences emerged for precision (respectively, $\sigma=75.75[73.76,77.600]$ and $74.02[71.62$, $76.24] ; \mathrm{M}=71.12$ and 71.52, $\mathrm{SD}=10.09$ and $11.42 ; \mathrm{t}_{28}=.18, \mathrm{p}=.86, \mathrm{BF}_{10}=0.67$ ) or guessing $\left(\lambda=6.6 \mathrm{e}-6\right.$ [9.8e-5, .013] and 3.9e-4 [1.8e-4, .015]; $\mathrm{M}=.020$ and $.016, \mathrm{SD}=.047$ and $.044 ; \mathrm{t}_{28}=$ $\left..27, \mathrm{p}=.79, \mathrm{BF}_{10}=0.67\right)$. However, bias was higher-such that recent responses were favored more-for high compared to low confidence (respectively, $\mu=10.41[8.17,12.81]$ and $3.39[.68$, 7.09]; $\mathrm{M}=13.24$ and -.016, $\mathrm{SD}=18.70$ and 17.77; $\mathrm{t}_{28}=3.73, \mathrm{p}<.001, \mathrm{BF}_{10}=16.12$; see Figure 2C). 


\section{Discussion}

In the current study, we adapted a recently-developed approach of assessing retrieval precision to the domain of temporal information, which is a ready target for investigation given its prevalent association with episodic memory and its ever-changing nature. By having subjects estimate the temporal position of encoding a series of pictures, and novelly applying a set of mixture models to the distributions of behavioral errors, we extend the findings of recent studies on temporal memory to test for the involvement of decision-making factors such as bias and guessing. Below, we discuss three key findings of our study and their implications for understanding episodic retrieval, noting in particular the relevant limitations of this initial investigation.

The finding most relevant to our main hypothesis was that temporal precision declined as memories became more remote. Recency effects in recognition memory, whereby responses tend to be quicker and more accurate for items presented closer to the time of test, have been well established for decades (e.g., Hintzman \& Block, 1971; Hintzman et al., 1973; Monsell, 1978). Early studies of this sort used shorter study lists and required subjects to make coarser temporal judgments, precluding a fine-grained understanding of how temporal information may degrade across time. Although our results replicate these prior findings, they also further our understanding of recency effects across longer study lists and in combination with newer continuous-report approaches to testing memory retrieval. This effect of worsening precision over time, parameterized as the standard deviation of a normal function fit to the error distribution, is especially notable given its reliability within the bounds of the 17.5-minute study list, both from our main analysis that divided the list in half - giving rise to an average remoterecent time difference of about 6 minutes, based on the midpoint of each half — and from a secondary, quartile-based analysis. While the latter analysis is less optimal in terms of trial numbers per bin, it afforded the ability to distinguish differences in precision between the middle and ends of the list from the remote-recent effect, which was evident even between the middle quartiles ( $\sim 3$ minutes between midpoints). For additional support, a hierarchical regression-based analysis confirmed that precision at the trial level decreased linearly with passing time (results reported online at https://osf.io/c7nge). Together, this evidence extends that of previous studies in which estimates of retrieval precision, for either continuous color (Brady et al., 2013) or spatial location (Harlow \& Donaldson, 2013; Lew et al., 2016; Sutterer et al., 2019), have been shown to decline with longer study-test delays. The convergence across these domains also informs a much more expansive literature on forgetting (for reviews, see Friedman, 1993; Wixted, 2004; Howard et al., 2015), further specifying the decline in accuracy with remoteness as diminished precision rather than increased guessing.

The involvement of random guessing, a non-mnemonic factor, in temporal judgments was tested via inclusion of a uniform distribution in the mixture modeling. We observed minimal influence of guessing of this sort, contrary to numerous prior findings (e.g., Zhang \& Luck, 2008; Brady et al., 2013; Harlow \& Donaldson, 2013; Harlow \& Yonelinas, 2016), which could be explained in multiple but likely related ways. On one hand, whereas guessing along circular representations of location and color has been successfully modeled this way, our subjects tended to respond less frequently near the ends of the study list, similar to previous observations with a timeline (Jenkins \& Ranganath, 2010) and near the edges of two-dimensional space (e.g., Nilakantan et al., 2018). Notably, in the spatial retrieval tasks of Nilakantan et al. (2017, 2018), 
guessing was evident (non-zero) even when considering the lower response rates at the edges of the display. On the other hand, an alternative explanation for the discrepancy is that, beyond any property of our response method, memory for time is inherently distinct from that for other features. That is, for any given memory, there could invariably be some temporal information that can be retrieved, albeit vague; for instance, it might always be possible to place a memory in the correct as opposed to incorrect half of a study list, even when precision is rather low. The nature of cognitive processes underlying these vague (i.e. acontextual) memories, as well as whether guessing is better captured by other (non-uniform) functions, are critical issues to address in future studies.

The final set of findings worth discussing are related to response bias. First, there was an overall tendency to judge study items as occurring more recently than they actually did. This finding is consistent with some studies of judgments of recency (e.g., Hintzman \& Block, 1971; McCormack, 1984), including a subset using absolute judgments that are analogous to our task (Hinrichs \& Buschke, 1968; Lockhart, 1969; Linton, 1975; Underwood, 1977). Notably, whereas the latter studies primarily tested memory over either short or very long periods (i.e. seconds vs. months/years), our design aligns better with the vast majority of modern studies of long-term memory, where study-test delay is typically on the order of tens of minutes. One finding that appears to stand in contrast to the recency bias shown here and in the studies noted above is from Hintzman et al. (1973). They asked subjects to coarsely judge whether items came from the beginning, middle, or end of a study list and reported a tendency to respond toward the middle of the list. Although bias seems to be taking a different form in the Hintzman et al. study, it is possible that the effect is more related to task demands rather than memory per se, possibly similar to our finding of the tendency to respond away from the ends of the list. In the context of modeling retrieval precision in long-term memory, though, the current study is to our knowledge novel in testing for response bias. A design such as this could thus provide an important bridge for integrating temporal judgments, as well as their associated biases, into existing episodic memory theories. The second finding was that temporal judgments made with high confidence were associated with an elevated level of recency bias compared to those made with low confidence. Admittedly, we had no strong predictions about how subjects might arrive at judging confidence. Whereas the instructions emphasized that it should be treated separately from precision (Harlow \& Yonelinas, 2016), it seemed possible that subjects would resort to relying on precision in the absence of any other evidence. Alternatively, the basis for confidence could have varied from subject to subject, resulting in the measure being just a noisier version of the precision judgment (also see Harlow \& Donaldson, 2013). Nonetheless, it is plausible that the bias effect we observed is due to some subjective factor, such as familiarity strength, that gives rise to increases in both confidence and a feeling of recency.

Just as recent methods of measuring retrieval precision have advanced working memory theories beyond a predominant focus on capacity (for reviews, see Cowan, 2001; Luck \& Vogel, 2013), they can conceivably move research on episodic memory past the "all-or-none", thresholded treatment of recollection (Wixted, 2007; Parks \& Yonelinas, 2009). The application of these methods to identify the subtle features of memory retrieval could be essential not only for interfacing behavioral measures with their underlying neurocognitive processes, which might also operate continuously (e.g., Johnson et al., 2009; Murray et al., 2015), but also for detecting early forms of memory decline before more substantial categorical effects become apparent. As we have demonstrated here, time has the potential to be a useful dimension for investigating such 
phenomena, given its ever-present association with events and its changing dynamics even within single-session laboratory tests of episodic memory. 


\section{References}

Andrieu, C., De Freitas, N., Doucet, A., \& Jordan, M. (2003). An introduction to MCMC for machine learning. Machine Learning, 50(1), 5-43. doi:10.1023/A:1020281327116

Brady, T. F., Konkle, T., Gill, J., Oliva, A., \& Alvarez, G. A. (2013). Visual long-term memory has the same limit on fidelity as visual working memory. Psychological Science, 24(6), 981-990. doi:10.1177/0956797612465439

Cowan, N. (2001). The magical number 4 in short-term memory: A reconsideration of mental storage capacity. Behavioral and Brain Sciences, 24(1), 87-114. doi:10.1017/S0140525X01003922

Eichenbaum, H. (2013). Memory on time. Trends in Cognitive Sciences, 17(2), 81-88. doi:10.1016/j.tics.2012.12.007

Friedman, W. J. (1993). Memory for the time of past events. Psychological Bulletin, 113(1), 4466. doi:10.1037/0033-2909.113.1.44

Gelman, A., \& Rubin, D. B. (1992). Inference from iterative simulation using multiple sequences. Statistical Science, 7(4), 457-472. doi:10.1214/ss\%2F1177011136

Harlow, I. M., \& Donaldson, D. I. (2013). Source accuracy data reveal the thresholded nature of human episodic memory. Psychonomic Bulletin \& Review, 20(2), 318-325. doi:10.3758/s13423-012-0340-9

Harlow, I. M., \& Yonelinas, A. P. (2016). Distinguishing between the success and precision of recollection. Memory, 24(1), 114-127. doi:10.1080/09658211.2014.988162

Hayes, S. M., Buchler, N., Stokes, J., Kragel, J., \& Cabeza, R. (2011). Neural correlates of confidence during item recognition and source memory retrieval: Evidence for both dualprocess and strength memory theories. Journal of Cognitive Neuroscience, 23(12), 39593971. doi:10.1162/jocn_a_00086

Hinrichs, J. V., \& Buschke, H. (1968). Judgment of recency under steady-state conditions. Journal of Experimental Psychology, 78(4, Pt. 1), 574-579. doi:10.1037/h0026615

Hintzman, D. L., \& Block, R. A. (1971). Repetition and memory: Evidence for a multiple-trace hypothesis. Journal of Experimental Psychology, 88(3), 297-306. doi:10.1037/h0030907

Hintzman, D. L., Block, R. A., \& Summers, J. J. (1973). Contextual associations and memory for serial position. Journal of Experimental Psychology, 97(2), 220-229. doi: $10.1037 / \mathrm{h} 0033884$

Howard, M. W. (2018). Memory as perception of the past: Compressed time in mind and brain. Trends in Cognitive Sciences, 22(2), 124-136. doi:10.1016/j.tics.2017.11.004

Howard, M. W., Shankar, K. H., Aue, W. R., \& Criss, A. H. (2015). A distributed representation of internal time. Psychological Review, 122(1), 24-53. doi:10.1037/a0037840

Jenkins, L. J., \& Ranganath, C. (2010). Prefrontal and medial temporal lobe activity at encoding predicts temporal context memory. Journal of Neuroscience, 30(46), 15558-15565. doi:10.1523/JNEUROSCI.1337-10.2010 
Johnson, J. D., McDuff, S. G. R., Rugg, M. D., \& Norman, K. A. (2009). Recollection, familiarity, and cortical reinstatement: A multivoxel pattern analysis. Neuron, 63(5), 697-708. doi:10.1016/j.neuron.2009.08.011

Lew, T. F., Pashler, H. E., \& Vul, E. (2016). Fragile associations coexist with robust memories for precise details in long-term memory. Journal of Experimental Psychology: Learning, Memory, and Cognition, 42(3), 379-393. doi:10.1037/xlm0000178

Linton, M. (1975). Memory for real-world events. In D. A. Norman, \& D. E. Rumelhart (Eds.), Exploration in Cognition (pp. 376-404). San Francisco: W. H. Freeman.

Lockhart, R. S. (1969). Recency discrimination predicted from absolute lag judgments. Perception \& Psychophysics, 6, 42-44. doi:10.3758/BF03210673

Lositsky, O., Chen, J., Toker, D., Honey, C. J., Shvartsman, M., Poppenk, J. L., Hasson, U., \& Norman, K. A. (2016). Neural pattern change during encoding of a narrative predicts retrospective duration estimates. eLife, e16070. doi:10.7554/eLife.16070

Luck, S. J., \& Vogel, E. K. (2013). Visual working memory capacity: From psychophysics and neurobiology to individual differences. Trends in Cognitive Sciences, 17(8), 391-400. doi:10.1016/j.tics.2013.06.006

Ma, W. J., Husain, M., \& Bays, P. M. (2014). Changing concepts of working memory. Nature Neuroscience, 17, 347-356. doi:10.1038/nn.3655

McCormack, P. D. (1984). Aging and recognition memory: Methodological and interpretive problems. Experimental Aging Research, 10(4), 215-219. doi:10.1080/03610738408258468

Mickes, L., Wais, P., \& Wixted, J. T. (2009). Recollection is a continuous process: Implications for dual-process theories of recognition memory. Psychological Science, 20(4), 509-515. doi:10.1111/j.1467-9280.2009.02324.X

Monsell, S. (1978). Recency, immediate recognition memory, and reaction time. Cognitive Psychology, 10(4), 465-501. doi:10.1016/0010-0285(78)90008-7

Montaldi, D., Spencer, T. J., Roberts, N., \& Mayes, A. R. (2006). The neural system that mediates familiarity memory. Hippocampus, 16(5), 504-520. doi:10.1002/hipo.20178

Montchal, M. E., Reagh, Z. M., \& Yassa, M. A. (2019). Precise temporal memories are supported by the lateral entorhinal cortex in humans. Nature Neuroscience, 22, 284-288. doi:10.1038/s41593-018-0303-1

Morey, R. D., Rouder, J. N., \& Jamil, T. (2018). BayesFactor: Computation of Bayes Factors for common designs. $R$ package version $0.9 .12-4.2$. https://CRAN.Rproject.org/package $=$ BayesFactor

Murray, J. G., Howie, C. A., \& Donaldson, D. I. (2015). The neural mechanism underlying recollection is sensitive to the quality of episodic memory: Event related potentials reveal a some-or-none threshold. NeuroImage, 120, 298-308.

doi:10.1016/j.neuroimage.2015.06.069 
Nilakantan, A. S., Bridge, D. J., Gagnon, E. P., VanHaerents, S. A., \& Voss, J. L. (2017). Stimulation of the Posterior Cortical-Hippocampal Network Enhances Precision of Memory Recollection. Current Biology, 27(3), 465-470. doi:10.1016/j.cub.2016.12.042

Nilakantan, A. S., Bridge, D. J., VanHaerents, S., \& Voss, J. L. (2018). Distinguishing the precision of spatial recollection from its success: Evidence from healthy aging and unilateral mesial temporal lobe resection. Neuropsychologia, 119, 101-106. doi:10.1016/j.neuropsychologia.2018.07.035

Parks, C. M., \& Yonelinas, A. P. (2009). Evidence for a memory threshold in second-choice recognition memory responses. Proceedings of the National Academy of Sciences of the United States of America, 106(28), 11515-11519. doi:10.1073/pnas.0905505106

Ranganath, C., \& Hseih, L. -T. (2016). The hippocampus: A special place for time. Annals of the New York Academy of Sciences, 1369(1), 93-110. doi:10.1111/nyas.13043

Richter, F. R., Cooper, R. A., Bays, P. M., \& Simons, J. S. (2016). Distinct neural mechanisms underlie the success, precision, and vividness of episodic memory. eLife, 5. doi:10.7554/eLife. 18260

Rouder, J. N., Speckman, P. L., Sun, D., Morey, R. D., \& Iverson, G. (2009). Bayesian t-tests for accepting and rejecting the null hypothesis. Psychonomic Bulletin \& Review, 16(2), 225237. doi:10.3758/PBR.16.2.225.

Schwarz, G. (1978). Estimating the dimension of a model. Annals of Statistics, 6(2), 461-464. doi:10.1214/aos/1176344136

Schwarzkopf, D. S. (2015). Bayes Factors MATLAB functions. Figshare. https://figshare.com/articles/Bayes_Factors_Matlab_functions/1357917/1. doi:10.6084/m9.figshare.1357917.v1

Skinner, E. I., \& Fernandes, M. A. (2007). Neural correlates of recollection and familiarity: A review of neuroimaging patient data. Neuropsychologia, 45(10), 2163-2179. doi:10.1016/j.neuropsychologia.2007.03.007

Suchow, J. W., Brady, T. F., Fougnie, D., \& Alvarez, G. A. (2013). Modeling visual working memory with the MemToolbox. Journal of Vision, 13(10):9, 1-8. doi:10.1167/13.10.9

Sutterer, D. W., Foster, J. J., Serences, J. T., Vogel, E. K., \& Awh, E. (2019). Alpha-band oscillations track the retrieval of precise spatial representations from long-term memory. Journal of Neurophysiology, 122(2), 539-551. doi:10.1152/jn.00268.2019

Underwood, B. J. (1977). Temporal codes for memories: Issues and problems. Hillsdale, NJ: Erlbaum.

Vilberg, K. L., \& Rugg, M. D. (2007). Dissociation of the neural correlates of recognition memory according to familiarity, recollection, and amount of recollected information. Neuropsychologia, 45(10), 2216-2225. doi:10.1016/j.neuropsychologia.2007.02.027

Wilding, E. (2000). In what way does the parietal ERP old/new effect index recollection? International Journal of Psychophysiology, 35(1), 81-87. doi:10.1016/s01678760(99)00095-1 
Wixted, J. T. (2004). The psychology and neuroscience of forgetting. Annual Review of Psychology, 55(1), 235-269. doi:10.1146/annurev.psych.55.090902.141555

Wixted, J. T. (2007). Dual-process theory and signal-detection theory of recognition memory. Psychological Review, 114(1), 152-176. doi:10.1037/0033-295X.114.1.152

Yonelinas, A. P. (2002). The nature of recollection and familiarity: A review of 30 years of research. Journal of Memory and Language, 46(3), 441-517. doi:10.1006/jmla.2002.2864

Yonelinas, A. P., Otten, L. J., Shaw, K. N., \& Rugg, M. D. (2005). Separating the brain regions involved in recollection and familiarity in recognition memory. Journal of Neuroscience, 25(11), 3002-3008. doi:10.1523/JNEUROSCI.5295-04.2005

Zhang, W., \& Luck, S. J. (2008). Discrete fixed-resolution representations in visual working memory. Nature, 453(7192), 233-235. doi:10.1038/nature06860

\section{Open Practices Statement}

The experimental data and analysis scripts are publicly available on the Open Science Framework (OSF) website at https://osf.io/c7nge. This experiment was not formally preregistered.

\section{Acknowledgments}

We thank Annika David, Brooklyn Hawkinson, Brittney Herrmann, Alyssa Labonte, Kirstin McRaith, and Mikella Vermaire for assistance with data collection. The authors declare no conflicts of interest. 